Check for updates

Cite this: Phys. Chem. Chem. Phys., 2021, 23, 14433

Received 19th February 2021, Accepted 4th June 2021

DOI: $10.1039 / \mathrm{d} 1 \mathrm{cp} 00779 \mathrm{c}$

rsc.li/pccp

\section{Hydration enthalpies of amorphous sucrose, trehalose and maltodextrins and their relationship with heat capacities $\dagger$}

\author{
Ekaterina Bogdanova, (D) ${ }^{\text {ab }}$ Anna Millqvist Fureby ${ }^{c}$ and Vitaly Kocherbitov (D) *ab
}

\begin{abstract}
The mechanisms of glass transitions and the behavior of small solute molecules in a glassy matrix are some of the most important topics of modern thermodynamics. Water plays an important role in the physical and chemical stability of lyophilized biologics formulations, in which glassy carbohydrates act as cryoprotectants and stabilizers. In this study, sorption calorimetry was used for simultaneous measurements of water activity and the enthalpy of water sorption by amorphous sucrose, trehalose and maltodextrins. Moreover, the heat capacity of these carbohydrates in mixtures with water was measured by DSC in a broad range of water contents. The hydration enthalpies of glassy sucrose, trehalose and maltodextrins are exothermic, and the enthalpy change of water-induced isothermal glass transitions is higher for small molecules. The partial molar enthalpy of mixing of water in slow experiments is about $-18 \mathrm{~kJ} \mathrm{~mol}{ }^{-1}$, but less exothermic in the case of small molecules at fast hydration scan rates. By measuring the heat capacities of disaccharides and maltodextrins as a function of water content, we separated the contributions of carbohydrates and water to the total heat capacities of the mixtures. The combination of these data allowed testing of thermodynamic models describing the hydration-induced glass transitions. The heat capacity changes calculated by the fitting of the hydration enthalpy data for disaccharides are in good agreement with the heat capacity data obtained by DSC, while for maltodextrins, the effect of sub- $T_{\mathrm{g}}$ transitions should be taken into account. Combining the data obtained by different techniques, we found a distinct difference in the behavior of water in glassy polymers compared to that in glassy disaccharides. By understanding the behavior of water in glassy carbohydrates, these results can be used to improve the design of freeze-dried formulations of proteins and probiotics.
\end{abstract}

\section{Introduction}

The study of glassy states is one of the key topics of current thermodynamics. Amorphous disaccharides and polysaccharides are of particular interest for pharmaceutical and food industries as these can stabilize proteins, peptides, antibodies and bacteria against degradation during the freeze-drying process and storage. ${ }^{1}$ One of the important properties of amorphous materials is the glass transition. Polymers and other amorphous materials are fragile and hard at temperatures much lower than the glass transition temperature (glassy state), and soft and flexible at temperatures much higher than the glass transition temperature

\footnotetext{
${ }^{a}$ Biomedical Science, Malmö University, SE-20506, Malmö, Sweden. E-mail: vitaly.kocherbitov@mau.se

${ }^{b}$ Biofilms research center for Biointerfaces, Malmö, Sweden

${ }^{c}$ RISE Research Institutes of Sweden, Stockholm, Sweden

$\dagger$ Electronic supplementary information (ESI) available: Detailed fitting results, additional heat capacity data and enthalpies of sub- $T_{\mathrm{g}}$ transitions in Glucidex as a function of water content. See DOI: $10.1039 / \mathrm{d} 1 \mathrm{cp} 00779 \mathrm{c}$
}

(rubbery or liquid state). The glass transition parameters are used for predicting the long-term stability of solid formulations ${ }^{2-4}$ and as a guidance for selecting storage conditions. ${ }^{1,5}$ Protein degradation increases above the glass transition temperature, ${ }^{3}$ since the molecular mobility is much higher in the rubbery state. Water is known to decrease the glass transition temperature and increase the molecular mobility, thus acting as a plasticizer. This behavior can be described by the empirical Gordon-Taylor equation ${ }^{6,7}$ which includes the glass transition temperatures of single components and a parameter individual for a particular binary system. There are, however, opposite examples, indicating that in confinement (and in certain cases in the bulk) water can act as antiplasticizer. $^{8-10}$

In the case of amorphous sugars, water sorption changes their physico-chemical properties from rigid amorphous solids to highly viscous liquids and then to solid crystals (if the substance is prone to crystallization) that are dissolved upon further addition of water. The most common technique to study moisture sorption is gravimetric vapor sorption, which 
measures sorption isotherms, i.e., plots of water activity versus water content at a constant temperature. The water sorption isotherms of amorphous carbohydrates and polymers can be analyzed using either surface adsorption or bulk mixing models. To the first group belong the well-known Langmuir adsorption model, Brunauer-Emmett-Teller (BET) model and Guggenheim-Anderson-de Boer (GAB) model. In this case, the hydration process considers a monolayer or multilayer physical adsorption of adsorbate molecules on a plane surface where all adsorption sites are equivalent, with or without interactions between layers. This approach is successfully used in materials science and catalysis, but biopolymers have important distinguishing features. In particular, water molecules are rather absorbed by the bulk of amorphous sugars than being adsorbed at the surface. ${ }^{11,12}$ As a result of the mismatch between the assumptions of the adsorption models and the physics of the absorption process, they are unable to account for significant hysteresis between the sorption and desorption isotherms in the case of biopolymers, as the models do not consider the plasticizing effect of water, as well as the swelling of the material. Alternatively, sorption of water can be described using models that assume a mixing in the bulk, rather than adsorption at a surface. In particular, the Flory-Huggins solution theory of polymers shows good agreement with the experimental data for the liquid or rubbery state. However, it is not applicable for the description of polymers in the glassy state. Vrentas and Vrentas ${ }^{13}$ combined the Flory-Huggins theory with the free volume theory and proposed a model that satisfactorily describes the characteristic shape of water sorption isotherms for polyvinylpyrrolidone (PVP), ${ }^{14}$ starch, ${ }^{15}$ and small molecules: glucose and trehalose $^{16}$ and amorphous mixtures of sugars (trehalose, sucrose) - PVP. ${ }^{17}$

In addition to water sorption isotherms, fundamental thermodynamic properties of matter such as heat capacity in the glassy state and enthalpy of mixing are used for understanding water-amorphous carbohydrate interactions. Glass is not a thermodynamically stable state, it is less ordered than crystalline state; this has an impact on its thermodynamic properties. It was shown ${ }^{18}$ that the heat capacity of the amorphous form of sucrose below $T_{\mathrm{g}}$ is significantly higher than that of crystals. Similar data were obtained for other pharmaceutically important glass-forming substances such as sorbitol, trehalose and indomethacin. ${ }^{18}$ Moreover, the heat capacity of an amorphous material is dependent on the thermal history of the sample. In particular, a lyophilized material has a higher heat capacity than a quench-cooled and then aged material. It has also been shown that the heat capacity of sucrose melt decreases with time. ${ }^{18}$ Since the amorphous system tends to equilibrium, the heat capacity shifts towards values typical for crystalline materials. ${ }^{19}$ In general, microscopic molecular motions define the macroscopic thermodynamic properties such as enthalpy and heat capacity. Although heat capacity measurements are routine experiments in adiabatic or scanning calorimetry, a molecular-level interpretation of the experimental heat capacity data of amorphous polymers and their mixtures with water is not a trivial task, but may require statistical thermodynamic calculations. ${ }^{20}$

One of the examples of a thermodynamic variable that describes mixing process is the enthalpy of solution. The enthalpy of solution is an integral property that describes mixing with water in one step, from the solid state to the liquid state. The experimental data of the heat of solution are available in the literature. ${ }^{21}$ Due to its integral nature, this parameter is however not suitable for describing the changes that gradually occur in an amorphous material upon hydration. Another thermodynamic property that describes the interactions with water is the partial molar enthalpy of mixing of water $\left(H_{\mathrm{w}}^{m}\right)$. It is defined as the change of enthalpy upon the addition of infinitely small amounts of water (normalized per added amount). This is a differential property and is suitable for the characterization of changes that occur upon gradual hydration of a substance. The heat of mixing is the total result of many different interactions between components and gives useful information about complex systems. Due to their technical complexity, calorimetric measurements of water vapor sorption using glassy carbohydrates are rarely found in the literature. As an example, one can mention the work by Lechuga-Ballesteros and coworkers who used a thermal activity monitor to study the interactions between amorphous saccharides and water. ${ }^{12}$ This methodology allows the measurements of sorption or mixing enthalpy as a function of relative humidity, but water contents in the samples cannot be determined, which presents a substantial limitation.

Another calorimetric approach that does not have these limitations is the method of sorption calorimetry developed by Wadsö and coworkers. ${ }^{22}$ It is a unique technique for the measurements of the partial molar enthalpy of mixing and the water sorption isotherms in one experiment. It was successfully used for the investigation of interactions of water with biopolymers such as cellulose, ${ }^{11}$ mucins,${ }^{23}$ hyaluronic acid, ${ }^{24}$ acidhydrolysed starch $^{25}$ and lysozymes. ${ }^{26}$ It was shown that the initial hydration of amorphous biopolymers is exothermic. Water-induced glass transitions can be observed as an endothermic step in the partial molar enthalpy of the mixing curve $^{24,25,27}$ similar to the heat capacity step in DSC experiments. Crystallization typically produces an exothermic peak in the hydration enthalpy curve; ${ }^{25}$ however, an endothermic crystallization peak was observed for hyaluronic acid. ${ }^{24}$

Although the hydration of carbohydrate polymers and sugarbased surfactants ${ }^{27}$ was studied by sorption calorimetry before, the corresponding studies of low-molecular-weight amorphous carbohydrates have not been, to the best of our knowledge, reported in the literature. Here we present a comparative study of the hydration of amorphous carbohydrates (sucrose, trehalose and maltodextrins) using sorption calorimetry. Moreover, we present the measurements of heat capacities of watercarbohydrate mixtures as a function of water content. Furthermore, using a recently developed theory that describes the enthalpy of water sorption using glassy materials, ${ }^{28}$ we analyze the relationship between the heat capacities and the hydration enthalpies in the glassy state. 


\section{Materials and methods}

\subsection{Materials}

Sucrose (CAS 57-50-1) and trehalose dihydrate (CAS 6138-23-4) were purchased from Sigma Aldrich. Glucidex12 and Glucidex9 are corn maltodextrins, with dextrose equivalents (DE) of 12 and 9, respectively, which were kindly provided by Roquette (France). Milli-Q purified water was used for all the experiments (ELGA, Purelab Flex). Glucidex9 and Glucidex12 powders were used as received or dried in a vacuum at room temperature.

\subsection{Methods}

2.2.1. Differential scanning calorimetry (DSC). DSC measurements were performed using a DSC 1 (Mettler Toledo, Switzerland). Temperature calibration and heat flow calibration were done using indium. The glass transition temperature and changes in heat capacities were determined using STARe Software by ISO standard (ISO 11357-2:1999), where the midpoint of the step is reported. An empty aluminum crucible was used as a reference.

In the case of dry samples, they were placed in $40 \mu \mathrm{m}$ aluminum pans, hermetically sealed in a nitrogen atmosphere with relative humidity of less than $5 \% \mathrm{RH}$. The pans were scanned at different temperature ranges and a heating rate of $10{ }^{\circ} \mathrm{C} \min ^{-1}$.

We used equilibration or interrupted equilibration of a dried material at various relative humidities in saturated salt solutions such as $\operatorname{LiCl}\left(a_{\mathrm{w}}=0.11\right), \mathrm{MgCl}_{2}\left(a_{\mathrm{w}}=0.33\right), \mathrm{Mg}\left(\mathrm{NO}_{3}\right)_{2}\left(a_{\mathrm{w}}=\right.$ $0.53), \mathrm{NaCl}\left(a_{\mathrm{w}}=0.75\right), \mathrm{KCl}\left(a_{\mathrm{w}}=0.84\right)$, and $\mathrm{K}_{2} \mathrm{SO}_{4}\left(a_{\mathrm{w}}=0.97\right)^{29}$ to obtain different water contents in solid samples. The samples with water contents of more than $30 \mathrm{wt} \%$ were prepared in aluminum pans by weighing crystalline sucrose, trehalose dehydrate or maltodextrin powder, adding the required amounts of liquid MQ water (ELGA, Purelab Flex) and sealing the pans.

Heat capacity measurements were performed using the following temperature program: ramp to $25{ }^{\circ} \mathrm{C}$, isothermal $5 \mathrm{~min}$, cooling $10{ }^{\circ} \mathrm{C} \mathrm{min}^{-1}$ to $-50{ }^{\circ} \mathrm{C}$, isothermal $5 \mathrm{~min}$, and heating $10{ }^{\circ} \mathrm{C} \min ^{-1}$ to $60{ }^{\circ} \mathrm{C}(\operatorname{method} 1)$ or to $100{ }^{\circ} \mathrm{C}(\operatorname{method} 2)$. The first test runs with freeze-dried sucrose were performed up to $100{ }^{\circ} \mathrm{C}$. Since the samples crystallize about $90-100{ }^{\circ} \mathrm{C}$, for further experiments with sucrose, a lower maximum temperature was applied. Method 1 was used for sucrose heat capacity measurements, method 2 for trehalose and both for maltodextrins. The run was performed 4 times for sucrose and trehalose, and once for maltodextrins. The DSC was calibrated with sapphire. From the obtained DSC data, the heat capacity was calculated using MATLAB (MathWorks, Inc).

2.2.2. Preparation of amorphous sugars by freeze-drying. Amorphous sugars were prepared by lyophilization from aqueous solutions using a freeze-dryer (Epsilon 2-4 LSCplus, Martin Christ GmbH, Germany). The samples with solid contents of $10 \mathrm{wt} \%$ were freeze-dried in $6 \mathrm{~mL}$ clear glass vials with a diameter of $22 \mathrm{~mm}$ (Schott, Germany) filled with $2 \mathrm{~mL}$ of solutions. The vials were loaded at room temperature. During freezing, the temperature was lowered to $-45{ }^{\circ} \mathrm{C}$ in 3 hours and held isothermally for 2 hours. Then, the shelf was heated to
Table 1 Parameters of fast and slow scan rate sorption calorimetric experiments

\begin{tabular}{|c|c|c|c|c|c|c|}
\hline \multirow{2}{*}{$\begin{array}{l}\text { Diameter } \\
\text { of the } \\
\text { tube, } \mathrm{mm}\end{array}$} & \multirow{2}{*}{$\begin{array}{l}P_{\max } \\
\mu \mathrm{W}\end{array}$} & \multicolumn{4}{|c|}{$\frac{\partial a_{\mathrm{w}}}{\partial t}\left(a_{\mathrm{w}}=0.4\right), \mathrm{h}^{-1}$} & \multirow{2}{*}{$\begin{array}{l}\text { Scan } \\
\text { rate }\end{array}$} \\
\hline & & Glucidex9 & Glucidex12 & Sucrose & Trehalose & \\
\hline 3.6 & 99.2 & 0.005 & 0.004 & 0.001 & 0.003 & Slow \\
\hline 9.1 & 909.2 & 0.02 & 0.02 & 0.01 & 0.015 & Fast \\
\hline
\end{tabular}

$4{ }^{\circ} \mathrm{C}$ and the chamber pressure was lowered to $0.1 \mathrm{mbar}$. The primary drying was done at a shelf temperature of $4{ }^{\circ} \mathrm{C}$ and a chamber pressure of 0.1 mbar for 16 hours. For secondary drying, the temperature was increased to $20{ }^{\circ} \mathrm{C}$ in 1 hour, and the chamber pressure was lowered to 0.01 mbar. After the ramp, the shelf was held isothermally for $3 \mathrm{~h}$. At the end of the freeze-drying cycle, the chamber was filled with dry nitrogen, sealed under a vacuum and stored in a freezer at $-20{ }^{\circ} \mathrm{C}$ until further analysis. No collapse was observed in the vials.

2.2.3. Sorption calorimetry. Sorption calorimetric experiments were conducted at $25{ }^{\circ} \mathrm{C}$ in a $28 \mathrm{~mm}$ two-chamber sorption calorimetric cell inserted in a double-twin microcalorimeter. The samples under study were placed in the upper chamber, and pure water was injected into the lower chamber. The thermal powers released in the two chambers were monitored simultaneously. The water activity in the sorption experiments was calculated from the thermal power of vaporization of water in the lower chamber as described in ref. 30. The partial molar enthalpy of mixing of water was calculated according to ref. 31.

Two different connecting tubes between the sorption and desorption chambers were used in this work in separate experiments. A narrow tube (diameter $3.6 \mathrm{~mm}$ ) and a large tube (diameter $9.1 \mathrm{~mm}$ ) were assembled for slow and fast scan experiments, respectively. The properties of the tube and the corresponding thermal power in maltodextrin experiments are listed in Table 1. The signal at the fast scan rate is ten times higher than that at the slow scan rate, analogous to the difference between the DSC experiments at $10{ }^{\circ} \mathrm{C} \min ^{-1}$ and $1{ }^{\circ} \mathrm{C} \mathrm{min}^{-1}$. Experiments were performed 3 times for sucrose and trehalose with both narrow and large tubes, and once for Glucidex 9 and Glucidex12 at the desired scan rate.

\section{Results and discussion}

\subsection{Isothermal sorption calorimetry of sucrose, trehalose and maltodextrin}

3.1.1. Sorption calorimetry of sucrose. The sorption isotherm and the corresponding hydration enthalpy for sucrose at $25{ }^{\circ} \mathrm{C}$ are shown in Fig. 1a and c. The data were obtained for slow and fast scan rates and the influence of the scan rates will be discussed later, but first, we follow the phase transformations occurring in amorphous sucrose during hydration using the fast scan experiment (blue curves in Fig. 1) as an example. The water activity has a positive slope followed by a straight vertical line corresponding to sucrose crystallization. Above $8 \mathrm{wt} \%$ 

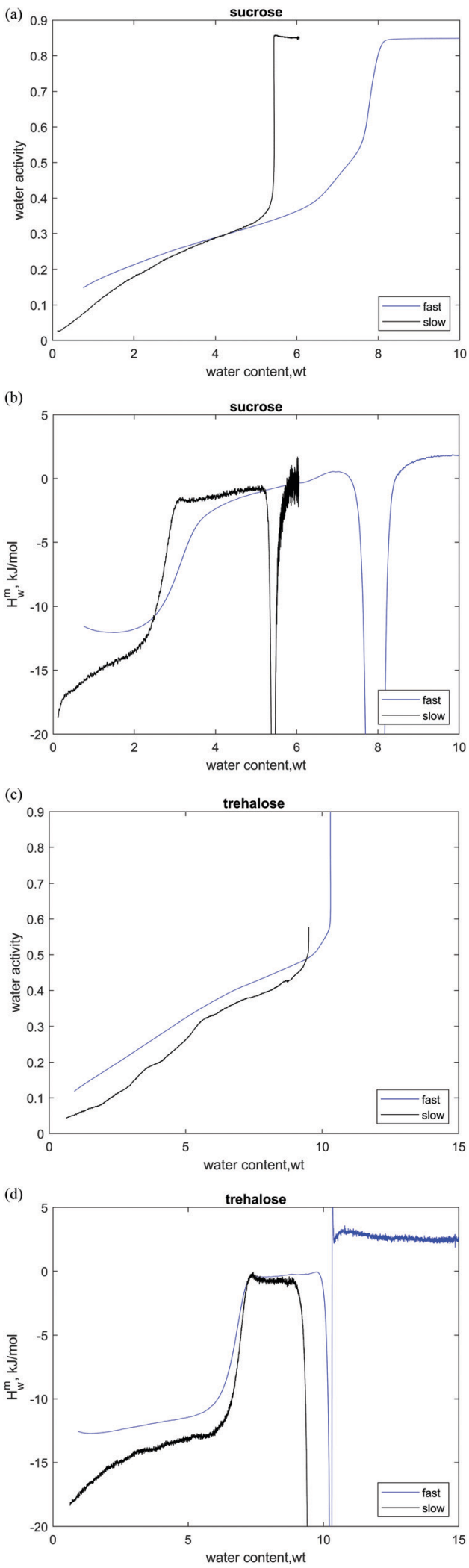

Fig. 1 Sorption isotherms of sucrose (a) and trehalose (c) and partial molar enthalpies of mixing of water for sucrose (b) and trehalose (d). The legend shows different water sorption rates, provided by different tube diameters (see the "Methods" section). of water, the water activity is constant, indicating a two-phase region (dissolution of crystals). The enthalpy curve (Fig. 1c) contains additional information related to the glass transition, which is not directly seen in the activity curve. In particular, the initial hydration of amorphous sucrose shows a highly exothermic $H_{\mathrm{w}}^{m}$ (approximately $-12 \mathrm{~kJ} \mathrm{~mol}^{-1}$ for the fast scan). Then, an endothermic thermal event in the form of a step in the region $2-3 \mathrm{wt} \%$ corresponding to a water-induced isothermal glass transition (Fig. 1c) is seen. This transition occurs at the water activity of about $0.2-0.3$ in agreement with previous studies. ${ }^{12,32}$ Next region is the mixing of metastable liquid with water and its heat effect is relatively close to 0 . Furthermore, an exothermic peak is observed, which corresponds to the hydration-induced crystallization of sucrose at about $8.0 \mathrm{wt} \%\left(a_{\mathrm{w}} \approx 0.5\right)$ in the case of the fast scan rate. The dissolution of crystals observed at higher water contents is accompanied by a relatively low endothermic effect.

The glassy system is not in a thermodynamically stable equilibrium state, hence it is not surprising that the slow scan rate profile for sucrose has substantial differences compared to the fast one (Fig. 1a and c). First, in the glassy state, the partial molar enthalpy of mixing of water is more exothermic for the slow scan rate $\left(-18 \mathrm{~kJ} \mathrm{~mol}^{-1}\right)$. Second, the glass transition is shifted to a somewhat lower hydration level, and third, crystallization occurs at much lower water content $(5.4 \mathrm{wt} \%$ vs. $8 \mathrm{wt} \%)$.

This scan rate dependence of the sorption behavior can be understood considering the kinetics of water sorption. For obtaining equilibrium thermodynamic data in sorption calorimetry, it is assumed that the resistance to water diffusion and the gradient of water concentration are present only in the vapor phase (between the vaporization chamber and the sorption chamber), while in the sample, there are no gradients of water activity. In the case of fast experiments when the vapor flow is high, and the water mobility in the glassy state is limited (and dependent on concentration ${ }^{33}$ ), this assumption is not fulfilled, and the gradients may appear in the sample. This leads to higher water activity on the surface of the sample in fast experiments, which is one of the reasons for the difference seen in Fig. 1a.

Crystallization of sucrose upon hydration is also dependent on the scan rates. In the fast experiment, one can see some signs of its beginning at $a_{\mathrm{w}}=0.4$, and at $a_{\mathrm{w}}=0.5$ it strongly accelerates. In the slow experiment, it starts around $a_{\mathrm{w}}=0.35$ and in terms of concentration, the scan rate dependence is even more obvious. The literature values for the threshold activity of crystallization are also diverse. For example, Lechuga-Ballesteros and coauthors detected sucrose crystallization using a thermal activity monitor at a water activity of $0.45{ }^{12}$ In an earlier work, it was shown that amorphous sucrose does not crystallize for 3 years at a water activity below 0.17 , but at 0.33 crystallization occurs within 24 hours. ${ }^{32}$ These results can be explained by the fact that crystallization of supercooled (or supersaturated) liquid is a spontaneous and stochastic process that can occur at a random point in time. Since the slow scans take a longer time than the fast ones, there is a higher probability that crystallization occurs at lower water contents. 
3.1.2. Sorption calorimetry of trehalose. The sorption isotherm and the hydration enthalpy for freeze-dried trehalose are presented in Fig. 1(b and d). The general shapes of the water activity and enthalpy curves for trehalose are similar to those observed for sucrose, but the transitions are shifted to higher water contents, which can be explained with a higher glass transition temperature of trehalose. Further discussion on the comparison of the sorption isotherms of sucrose and trehalose is presented in Section 3.4.

The initial hydration of amorphous trehalose shows a highly exothermic effect of about $-18 \mathrm{~kJ} \mathrm{~mol}^{-1}$ in the slow scan rate experiment and $-12 \mathrm{~kJ} \mathrm{~mol}^{-1}$ in the fast scan rate experiment. The water-induced glass transition takes place in the range of $6.5-7 \mathrm{wt} \%$, with $a_{\mathrm{w}}=0.35-0.4$ in both experiments, followed by the hydration of the metastable liquid with the heat effect close to zero. Crystallization occurs below $10 \mathrm{wt} \%$ in the slow case and slightly above in the fast case at $a_{\mathrm{w}}=0.45-0.5$. This crystallization event is consistent with the formation of trehalose dihydrate since the water content in this event is close to that in the trehalose dihydrate (9.52 wt\%). This is in good agreement with literature data, for example, crystallization of trehalose dihydrate was observed in ref. 14 in gravimetric water sorption experiments at $30{ }^{\circ} \mathrm{C}$ and $a_{\mathrm{w}}=0.4$.

According to the sorption isotherms of trehalose, the system contains more water at similar water activities in slow experiments, which can be attributed to the slow diffusion of water. As one can see from the comparison of the hydration plots for sucrose and trehalose, the scan rate has a stronger influence on the hydration profile of the former, which is especially clear in the case of crystallization. In this comparison, one should keep in mind that trehalose crystallizes in the form of a dihydrate, while sucrose crystallizes in its anhydrous form, which might influence the kinetics of crystallization.

3.1.3. Sorption calorimetry of maltodextrin. Sorption calorimetry data of Glucidex12 and Glucidex9 are presented in Fig. 2 which show similar behavior with previous experiments on acid hydrolyzed potato starch. ${ }^{25}$ The step at $20 \mathrm{wt} \%$ of water on the sorption isotherms corresponds to crystallization. The hydration enthalpy curves have positive slopes at a water content of less than $10 \mathrm{wt} \%$, for both maltodextrins. The waterinduced glass transition in Glucidex12 occurs at $13 \mathrm{wt} \%$ of water $\left(a_{\mathrm{w}}=0.7\right)$, and in Glucidex9 at $14 \mathrm{wt} \%\left(a_{\mathrm{w}}=0.8\right)$. This difference can be explained by the fact that Glucidex9 has an average molecular weight of $2000 \mathrm{~g} \mathrm{~mol}^{-1}$ while Glucidex has 12-1500 $\mathrm{g} \mathrm{mol}^{-1}$. The polymer with a higher average molecular weight (Glucidex9) undergoes water-induced glass transitions at higher water contents and higher water activities. This corresponds to the increase of $T_{\mathrm{g}}$ values in DSC experiments (see Table 2). Sharp exothermic peaks at $20 \mathrm{wt} \%$ of water indicate the crystallization of both Glucidex products, corresponding to the small steps in sorption isotherms at $20 \mathrm{wt} \%$ of water $\left(a_{\mathrm{w}}=0.9\right)$.

Neither the sorption isotherm of maltodextrin, nor its hydration enthalpy, shows a clear dependence on the humidity scan rate. This behavior is strikingly different compared to that of the sucrose and trehalose cases. We suggest that the primary
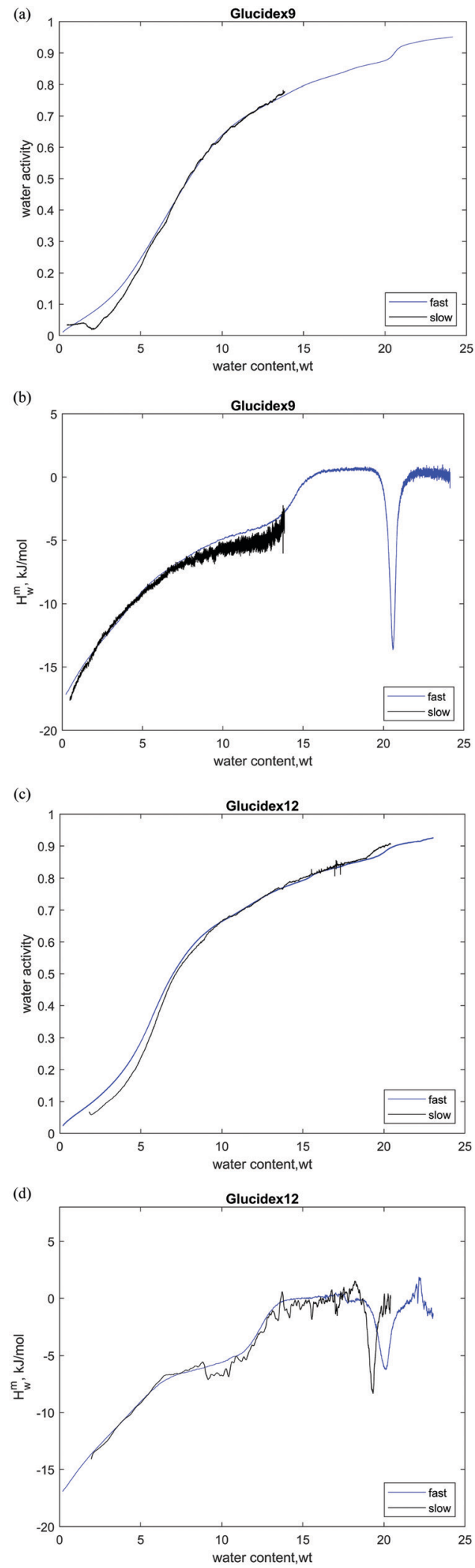

Fig. 2 Sorption isotherms of Glucidex9 (a) and Glucidex12 (c) and partial molar enthalpies of Glucidex9 (b) and Glucidex12 (d) and mixing of water as a function of water content. Different colors correspond to different tubes used in the sorption experiments. 
Table 2 The hydration enthalpy step at the glass transition temperature calculated using the Vrentas and Vrentas model (eqn (2)) in comparison with the experimental data. $A=-\frac{\partial T_{\mathrm{gm}}}{\partial \omega_{1}}$ was calculated at the point of the water-induced glass transition

\begin{tabular}{|c|c|c|c|c|c|c|}
\hline Substance & Scan rate & $\begin{array}{l}T_{\mathrm{g} 2} \\
(\mathrm{~K})\end{array}$ & $\begin{array}{l}\Delta C_{p 2} \\
\left(\mathrm{~J} \mathrm{~g}^{-1} \mathrm{~K}^{-1}\right)\end{array}$ & $A(\mathrm{~K})$ & $\begin{array}{l}\Delta H_{\mathrm{w}}^{m}\left(\mathrm{~kJ} \mathrm{~mol}^{-1}\right), \\
\text { eqn }(2)\end{array}$ & $\begin{array}{l}\Delta H_{\mathrm{w}}^{m}\left(\mathrm{~kJ} \mathrm{~mol}^{-1}\right) \\
\text { experiment }^{c}\end{array}$ \\
\hline Sucrose & Fast & 343 & 0.620 & 901 & 10 & 9.5 \\
\hline Sucrose & Slow & 343 & 0.620 & 901 & 10 & 11 \\
\hline Trehalose & Slow & 393 & 0.720 & 1034 & 13 & 11.5 \\
\hline Glucidex12 & Fast/slow & $473^{a}$ & $0.225^{b}$ & 1312 & 5.3 & 5 \\
\hline Glucidex9 & Fast & $498^{a}$ & $0.224^{b}$ & 1343 & 5.4 & 5 \\
\hline
\end{tabular}

${ }^{a}$ Data provided by the supplier. ${ }^{b}$ Data obtained by linear extrapolation. ${ }^{c}$ The experimental values are taken as a height of the glass transition step on the plot of the partial enthalpy of mixing.

reason for this is the difference in water diffusion properties. In particular, the water diffusion coefficient for the sucrose-water mixture is $10^{-16} \mathrm{~m}^{2} \mathrm{~s}^{-1}, 33$ while the water diffusion coefficient for the maltodextrin-water mixture was estimated to be $10^{-13} \mathrm{~m}^{2} \mathrm{~s}^{-1}$. ${ }^{34}$ In other words, water molecules in the polymer-water matrix move faster by three orders of magnitude compared to disaccharides and thus are distributed more evenly in the material, hence the absence of the effect of the scan rate for maltodextrins. In the case of sucrose and trehalose, in fast scans, the slow water diffusion can result in the formation of concentration gradients in the solid particles and variation in water uptake.

Water sorption isotherms of amorphous disaccharides, maltodextrins and starch materials have positive slopes. Upon crystallization, water activity changes its behavior and typically, a step on the activity curve is observed. Hence, it is easy to determine the critical water activity of the crystallization from the water sorption isotherm. However, the position of the isothermal water-induced glass transition is not clearly seen on the sorption isotherm. Simultaneous measurements of the sorption isotherm of the sample and the corresponding differential enthalpies of hydration make glass transition and crystallization phenomena clearly visible (Fig. 1 and 2).

\subsection{Models describing the enthalpy of sorption}

3.2.1. Earlier models (constant enthalpy models). A theory of mixing proposed by Vrentas and Vrentas ${ }^{13}$ predicts the values for the molar heat of sorption of the penetrant (in our case water) in the glassy polymer:

$$
H_{1}^{s}=-\frac{R T^{2}}{p_{1}^{0}} \frac{\partial p_{1}^{0}}{\partial T}-R T^{2} \frac{\partial \chi}{\partial T}-M_{1} \Delta C_{p 2}^{0} A
$$

where $M_{1}$ is the water molecular weight, $\Delta C_{p 2}^{0}$ is the heat capacity change of the polymer $\left(\mathrm{J} \mathrm{g}^{-1} \mathrm{~K}^{-1}\right)$, and $A$ is a positive coefficient characterizing the plasticizing effect of water and defined as $T_{\mathrm{gm}}=T_{\mathrm{g} 2}-A \omega_{1}$. In a more general sense, it is the derivative of the glass transition temperature on the composition which is $=-\frac{\partial T_{\mathrm{gm}}}{\partial \omega 1}$. Here and below, we use the following notation: $T_{\mathrm{g} 1}$ - glass transition temperature of pure water, $T_{\mathrm{g} 2}$ - glass transition temperature of the polymer, $\kappa$ - GordonTaylor parameter, $T_{\mathrm{gm}}$ - glass transition temperature of the mixture, $w_{1}$ - mass fraction of water, and $w_{2}$ - mass fraction of the polymer.

The exothermic contribution to the enthalpy of mixing due to the glassy state is described by the third term of eqn (1):

$$
\Delta H_{1}^{m}=-M_{1} \Delta C_{p 2}^{0} A
$$

where $\Delta C_{p 2}^{0}$ is taken from the DSC measurements, and $T_{\mathrm{g}}$ is experimentally available. Besides, for the calculation of the endothermic glass transition step in the mixing enthalpy curve, Kocherbitov and Söderman ${ }^{27}$ proposed the following equation:

$$
\Delta H_{1}^{m} r_{\mathrm{g}}=\Delta C_{p 2}^{0}\left(T_{\mathrm{g} 2}-T\right)
$$

where $r_{\mathrm{g}}$ is the water-to-carbohydrate mole ratio at the glass transition temperature and $\Delta C_{p 2}^{0}$ is expressed in $\mathrm{J} \mathrm{mol}^{-1} \mathrm{~K}^{-1}$. Eqn (2) and (3) assume a constant (not dependent on composition) contribution to $H_{1}^{m}$ from the glassy state, i.e., the hydration enthalpy in the glassy state is expected to be constant. The experimental data presented here show that this is not the case, especially for maltodextrin. Still, this approach can be used for an approximate estimation of the step of the hydration enthalpy $\left(\Delta H_{1}^{m}\right)$ at the glass transition temperature, which is demonstrated in Table 2. For the calculations, the $\Delta C_{p 2}^{0}$ values are taken from the DSC heating scans as steps on the heat capacity curves. The concentrations of the water-induced glass transitions are taken from the experimental plots of the enthalpy of mixing. The dependence of the glass transition temperature on water content and its derivative, $\frac{\partial T_{\mathrm{gm}}}{\partial \omega_{1}}$, is calculated from the Gordon-Taylor equation (kappa parameters are listed in Table 3). Despite the assumptions mentioned above, the calculated values are in reasonable agreement with the experimental data. Interestingly, despite the fact that in the dry limit the exothermic heat effect for maltodextrins (Fig. 2c and d) is similar or stronger than that for small sugars (Fig. 1c and d), the step in the enthalpy of mixing at the glass transition point is much smaller.

3.2.2. An extended model. The models mentioned above assume that the $H_{\mathrm{w}}^{m}$ of the glassy polymer with water is independent on water content. In other words, one can expect a straight horizontal line on the plot of enthalpy of mixing versus water content below the glass transition temperature. On the molecular level, it implies that the interactions between water and the glassy polymer matrix do not change upon 
Table 3 The heat capacity changes calculated by the fitting of the sorption calorimetry data, according to eqn (4). The heat capacity changes at $25^{\circ} \mathrm{C}$ obtained from the DSC data as described in Section 3.3 are shown for comparison

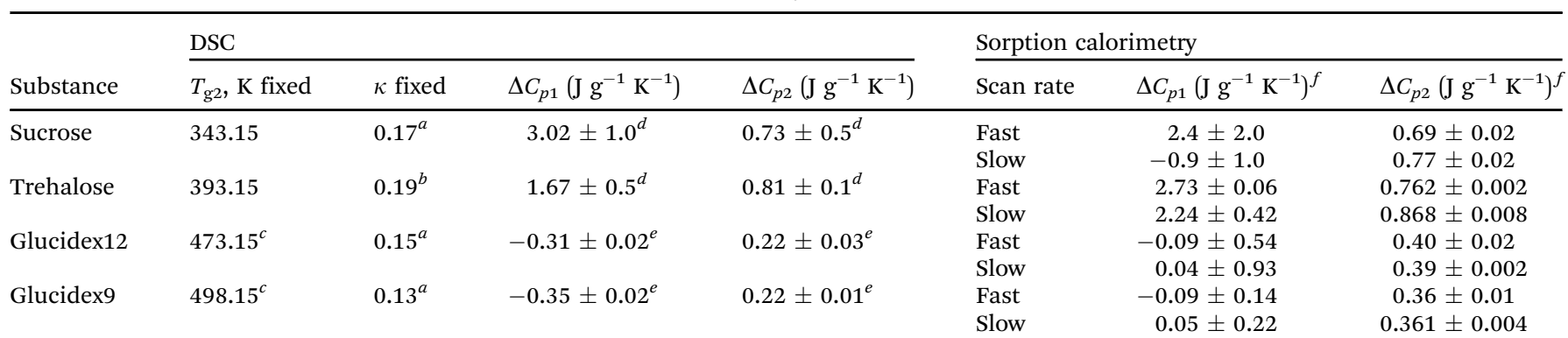

${ }^{a}$ Data from ref. $7 .{ }^{b}$ Data from ref. $36 .{ }^{c}$ Data provided by the supplier, obtained from the Gordon-Taylor equation. ${ }^{d}$ Obtained from a linear approximation of difference of heat capacities of liquid and glass at $25{ }^{\circ} \mathrm{C} .{ }^{e}$ Correction on sub-T according to eqn (8). ${ }^{f}$ The mean values of heat capacity changes obtained by fitting (with the standard deviations).

hydration. However, most often, the experimental data exhibit a slope and a concave-like feature in the enthalpy curves (Fig. 1c, $\mathrm{d}$ and $2 \mathrm{c}, \mathrm{d}$ ). The dependency of the partial molar enthalpy on water content (eqn (4)) obtained in the sorption calorimetry experiment can be described using a model recently proposed by our group. ${ }^{28}$ The model is based on a careful examination of the thermodynamic cycle of glass transition and uses certain approximations such as the Gordon-Taylor equation for the dependency of $T_{\mathrm{g}}$ on water content.

$$
\begin{aligned}
\frac{\Delta H_{1}^{m}}{M_{1}}= & \Delta C_{p 1}^{0}\left(T_{\mathrm{gm}}-T\right) \\
& -\frac{w_{1}\left(T_{\mathrm{g} 2}-T_{\mathrm{g} 1}\right)\left(w_{1} \Delta C_{p 1}^{0}\left(T_{\mathrm{gm}}\right)+w_{2} \Delta C_{p 2}^{0}\left(T_{\mathrm{gm}}\right)\right.}{\kappa\left(1+\left(\kappa^{-1}-1\right) w_{1}\right)^{2}}
\end{aligned}
$$

where $\Delta H_{1}^{m}$ is the partial molar enthalpy of mixing, $M_{1}$ is the water molecular weight, $\Delta C_{p 1}^{0}$ is the heat capacity change of the glassy water, $\Delta C_{p 2}^{0}$ is the heat capacity change of the polymer, $T_{\mathrm{gm}}$ is the glass transition temperature of the mixture, $w_{1}$ is the mass fraction of water, and $T$ is the temperature of the experiment. Eqn (4) has five parameters that can be either determined from fitting or fixed as constants known from other experiments: $T_{\mathrm{g} 1}, T_{\mathrm{g} 2}, \Delta C_{p 1}^{0}, \Delta C_{p 2}^{0}$ and $\kappa$. The glass transition temperature of water $\left(T_{\mathrm{g} 1}=-137^{\circ} \mathrm{C}\right)^{35}$ and the glass transition temperature of the dry polymer (when known) should be kept constant to obtain more stable fitting results. In this work, the glass transition temperature of pure water is taken as $-137{ }^{\circ} \mathrm{C}$ (the widely accepted value), commonly used for the GordonTaylor fits for water-amorphous sugar mixtures. ${ }^{36}$ We note, however, that an alternative interpretation suggests that the thermal event at $-137{ }^{\circ} \mathrm{C}$ is due to the unfreezing of local molecular motions and does not represent the glass-liquid transition. ${ }^{37}$

Earlier, the model was tested on starch and cellulose experimental data and gave a satisfactory prediction of the heat capacity change of the dry polymer and the kappa parameter. ${ }^{28}$ Here, the hydration enthalpy data of the glassy region of sucrose, trehalose, Glucidex12 and Glucidex9 were fitted with eqn (4) using MATLAB (MathWorks, Inc). The fitting results are presented in Table 3.

To check the fitting reproducibility, it was performed 3 times for different concentration ranges for every enthalpy curve.
Each curve was split into two parts: low water content and high water content (an example is presented in Fig. 3), where the concentration ranges are $0.01-0.04$ and $0.05-0.1$, respectively. The concentration ranges were different for each experiment, since the position of the water-induced glass transition varies from sample to sample. The fitting procedure was performed on the two data sets and for the whole glassy region (more data are shown in the ESI, $\uparrow$ Tables S1-S7).

The parameters $\left(\Delta C_{p 2}\right)$ obtained in fitting (sorption calorimetry columns in Table 3 ) for the fast and slow scan rates are

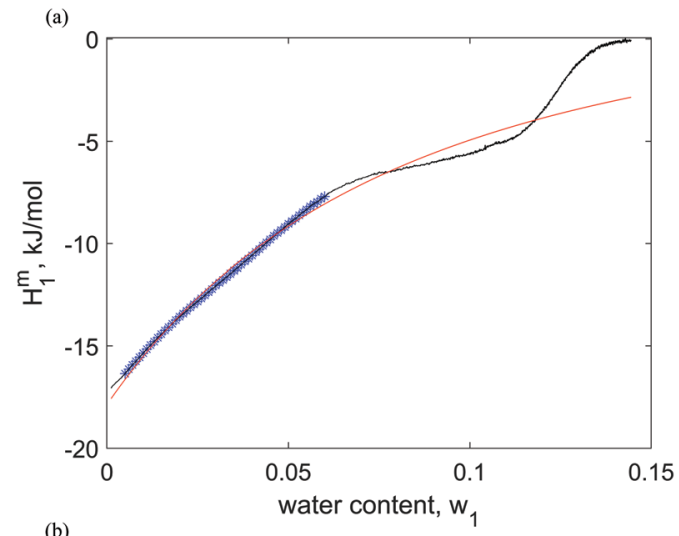

(b)

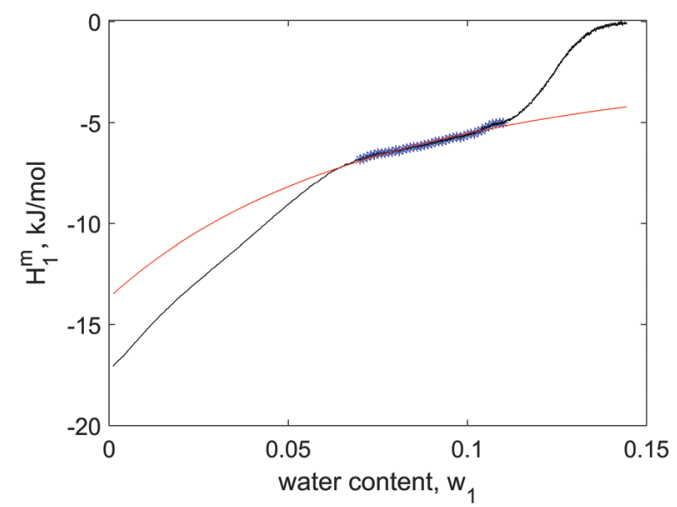

Fig. 3 Fitting results of eqn (3) for Glucidex12 for low (a) and high (b) water content range. Black curve - experimental data, blue - data points chosen for fitting, and red - resulted fitting. 
similar (about 10\% difference), show only few percent standard deviations, and are in good agreement with DSC data. In contrast, the heat capacity increment for water $\left(\Delta C_{p 1}\right)$ obtained in fitting exhibits larger standard deviations reflecting the differences in the thermodynamic parameters of water at different water contents. Moreover, the $\Delta C_{p 1}$ shows rather diverse values depending on the material and experimental conditions, which can be a valuable source of information on the properties of the glassy state. In particular, in the case of trehalose, $\Delta C_{p 1}$ is positive and shows values comparable to those observed for pure water. ${ }^{38}$ In the case of sucrose, this parameter is positive for the fast scan rate but negative for the slow experiment. One should, however, note that the fitting quality in the case of sucrose can be lower than that of the other three substances considered in this study due to a very narrow glassy region (see Fig. 1c). The initial disturbance of the calorimetric experiment in the case of fast scan rate might affect the quality of the experimental data. Moreover, the possible residual moisture that could not be fully removed from the freeze-dried samples can have a stronger influence on the results when the glassy region is narrow. The difference between the slow and fast scan rates can also appear due to the enthalpy relaxation. While in DSC experiments the enthalpy relaxation might be seen as a small endothermic peak after the glass transition step on the DSC heating curve, ${ }^{39}$ in sorption calorimetric experiments, it may influence the $H_{1}^{m}$ values in the glassy state.

For both Glucidex9 and Glucidex12, the heat capacity change of water is close to zero, which indicates a different state of water in the glassy maltodextrin compared to that in the sucrose and trehalose.

\subsection{The heat capacity and the temperature-induced glass transition}

3.3.1. The heat capacity of dry sucrose and trehalose. The heat capacity is the fundamental physical property, which characterizes the molecular motion of a system. The glass transition-induced heat capacity step $\left(\Delta C_{p}\right)$ is a key property of a two-component glassy system. Moreover, using it in combination with the Gordon-Taylor equation, one can calculate the enthalpy of mixing of water with glassy carbohydrates. In this section, we discuss our experimental DSC data on the heat capacity and the heat capacity step of sucrose, trehalose and maltodextrins and compare them with literature data.

Sucrose is a crystalline substance that needs to undergo a certain treatment in order to be obtained in an amorphous form. Often it is made amorphous by melting and quenchcooling. Below we compare the properties of amorphous sucrose obtained by quench-cooling and freeze-drying. The thermal behaviors of sucrose samples obtained by these two methods are presented in Fig. 4. In the experiment with melting, sucrose crystals were melted in a DSC pan using a temperature ramp of $20{ }^{\circ} \mathrm{C} \min ^{-1}$ to $186{ }^{\circ} \mathrm{C}$ and then quench-cooled by $20{ }^{\circ} \mathrm{C} \mathrm{min}^{-1}$ straight after. In the second scan (blue curve), there is a glass transition with $T_{\mathrm{g}}$ (midpoint) = $68.44{ }^{\circ} \mathrm{C}$ and $\Delta C_{p}=0.645 \mathrm{~J} \mathrm{~g}^{-1} \mathrm{~K}^{-1}$. The freeze-dried

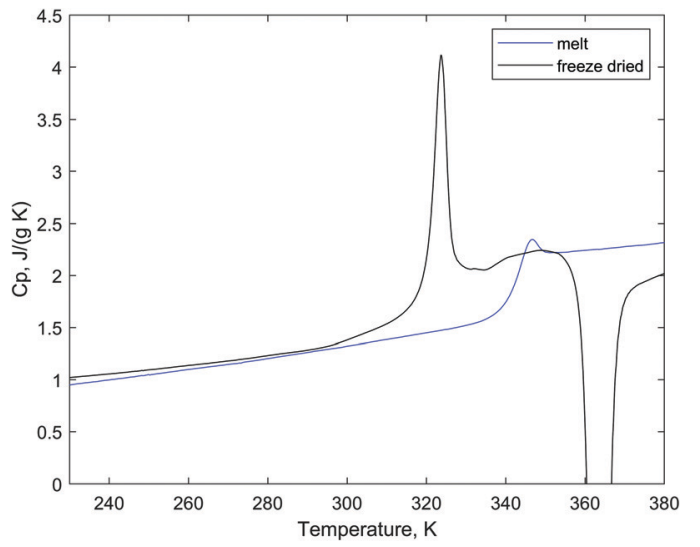

Fig. 4 DSC scans of amorphous sucrose with different thermal histories. The blue curve is the second scan of crystalline sucrose, and the black curve is the first scan of the freeze-dried amorphous material. The scan rate is $10{ }^{\circ} \mathrm{C} \mathrm{min}-1$.

amorphous sucrose (black curve) undergoes a glass transition at $70.60{ }^{\circ} \mathrm{C}$ with $\Delta C_{p}=0.762 \mathrm{~J} \mathrm{~g}^{-1} \mathrm{~K}^{-1}$ followed by a crystallization exotherm at about $110{ }^{\circ} \mathrm{C}$ and a broad melting (this part of the curve is not shown). It should be noted that all the DSC curves of the freeze-dried sucrose show crystallization at higher temperatures, while the quench-cooled samples do not. We suggest that the reason for such a behavior is the caramelization of the material during heating. After the second scan, the DSC pan with the melted sugar was opened; the obtained sample was transparent and yellow in color. A similar observation was made previously. ${ }^{40}$ Since the freeze-dried material was not treated at high temperatures, the pan contained only a white colored material after the first scan and even after the fourth DSC scan. Other factors, for example, higher surface area in the freeze-dried material, which increase the probability of surface-induced nucleation might affect the crystallization kinetics. Still, based on the color-change observation, for further experiments, only freeze-dried sucrose and trehalose were used.

The DSC curves of the freeze-dried sucrose show notable deviation from the baseline prior to the glass transition step, which might be described as an endothermic sub- $T_{\mathrm{g}}$ event. Sub- $T_{\mathrm{g}}$ endotherms (in the form of endothermic peaks) are wellknown for carbohydrate polymers, for example, starch-like materials. $^{25}$ Recently, Johari proposed that sucrose melt has this feature in DSC scans as well. ${ }^{41}$ The heat capacity of sucrose in the glassy state was reported to be between 1.31 and $1.38 \mathrm{~J} \mathrm{~g}^{-1} \mathrm{~K}^{-1}\left(25{ }^{\circ} \mathrm{C}\right),{ }^{18,19}$ whereas our measurements provided the values of $1.29 \pm 0.15 \mathrm{~J} \mathrm{~g}^{-1} \mathrm{~K}^{-1}$.

The DSC scan of the freeze-dried trehalose shows a glass transition step at $118{ }^{\circ} \mathrm{C}$ with $\Delta C_{p}=0.770 \mathrm{~J} \mathrm{~g}^{-1} \mathrm{~K}^{-1}$, and the heat capacity of the dry amorphous trehalose was measured to be $1.42 \mathrm{~J} \pm 0.10 \mathrm{~g}^{-1} \mathrm{~K}^{-1}$ at $25{ }^{\circ} \mathrm{C}$, which is in perfect agreement with the value from literature data $\left(1.43 \mathrm{~J} \mathrm{~g}^{-1} \mathrm{~K}^{-1}\right) .{ }^{18}$

3.3.2. The heat capacity of dry maltodextrins. The heat capacity of dry Glucidex 9 at $25{ }^{\circ} \mathrm{C}$ measured in this work is $1.09 \pm$ $0.08 \mathrm{~J} \mathrm{~g}^{-1} \mathrm{~K}^{-1}$, and Glucidex 12 at $25{ }^{\circ} \mathrm{C}$ is $1.11 \pm 0.09 \mathrm{~J} \mathrm{~g}^{-1} \mathrm{~K}^{-1}$, 
which are comparable with the literature values for glassy amylopectin $\left(1.16 \mathrm{~J} \mathrm{~g}^{-1} \mathrm{~K}^{-1}\right.$ from ref. 42 and $1.18 \mathrm{~J} \mathrm{~g}^{-1} \mathrm{~K}^{-1}$ from ref. 19) and for dry starch $\left(1.2 \mathrm{~J} \mathrm{~g}^{-1} \mathrm{~K}^{-1}\right.$, value graphically taken from ref. 20). The measurements of glass transition temperature and heat capacity change of dry maltodextrins in a conventional DSC instrument is a challenging task due to the chemical degradation of the material. The DSC signal of a heating ramp of $20^{\circ} \mathrm{C} \mathrm{min}^{-1}$ from 25 to $240{ }^{\circ} \mathrm{C}$ in hermetically sealed pans shows only one endothermic peak associated with the opening of the sealed lid due to the high pressure in the sample. The material turns brown after the DSC run. Therefore, the glass transition temperature of the dry amorphous maltodextrin was obtained by using the Gordon-Taylor approximation and was taken from the supplier of the material.

3.3.3. The heat capacities of mixtures with water. The heat capacities of the sucrose-water mixtures are presented in Fig. 5. The first scans of the freeze-dried materials differ from the subsequent scans. A possible reason for this is the compression of the fluffy freeze-dried powder after the first scan, which exhibits better thermal contact with the DSC pan and also enthalpy relaxation, which is more pronounced in the first scan (see Fig. S1 in the ESI $\dagger$ ). Each presented curve is the mean of the second, third and fourth runs of the freeze-dried material. The glass transition step with an enthalpy relaxation peak is clearly seen in the scans of the samples with water contents of 2.6-3.6 wt\%. The glass transition temperature, as expected, increases with the decreasing water content. Samples with water contents of $0.3-0.8$ wt $\%$ undergo glass transitions at temperatures outside the temperature program range, and only the beginning of the step can be seen on the plot. The sucrosewater system has a notable feature of exothermic deviation from the baseline prior to the glass transition step, which is also seen in the dry substance (a sub- $T_{\mathrm{g}}$ event as was noted above ${ }^{41}$ ). The heat capacity curves of the samples, which are liquids at $25{ }^{\circ} \mathrm{C}$, are presented in Fig. S2 in the ESI. $\dagger$ Samples with water contents of 19 and $25 \mathrm{wt} \%$ show glass transitions at low temperatures, and crystallization is not observed (neither for ice nor sucrose). The heat capacity scans of the samples with water contents of 40-90 wt\% exhibit glass transitions of freeze-concentrated solution $\left(T_{\mathrm{g}}^{\prime}\right)$ and ice melting events (Fig. S2 in the ESI $\dagger$ ).

The results of the heat capacity measurements of the trehalose-water mixtures are presented in Fig. 5b. The glassy trehalose-water samples with water contents of $3.4-5.1 \mathrm{wt} \%$ undergo glass transitions with a small but clear enthalpy relaxation event directly after the glass transition step. The glass transition temperature decreases with the increase of water content. The sample with $1 \mathrm{wt} \%$ water content undergoes glass transition at a higher temperature than the presented range. There are some deviations from the baseline (for the sample with $5.1 \mathrm{wt} \%$ ) prior to the glass transition step that might be a sign of a small sub- $T_{\mathrm{g}}$ event. The heat capacity curves of the samples with water contents higher than $60 \mathrm{wt} \%$ exhibit $T_{\mathrm{g}}^{\prime}$ and ice melting events and are presented in Fig. S3 in the ESI. $\dagger$

The heat capacity measurements of the Glucidex9-water mixtures are presented in Fig. $5 \mathrm{c}$. The heat capacity of the dry

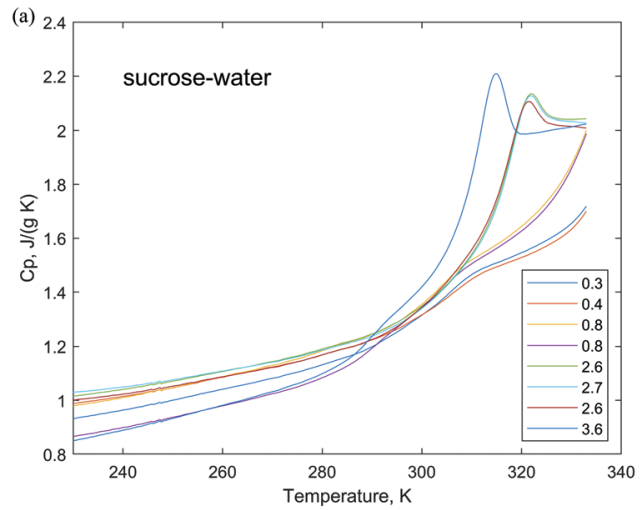

(b)

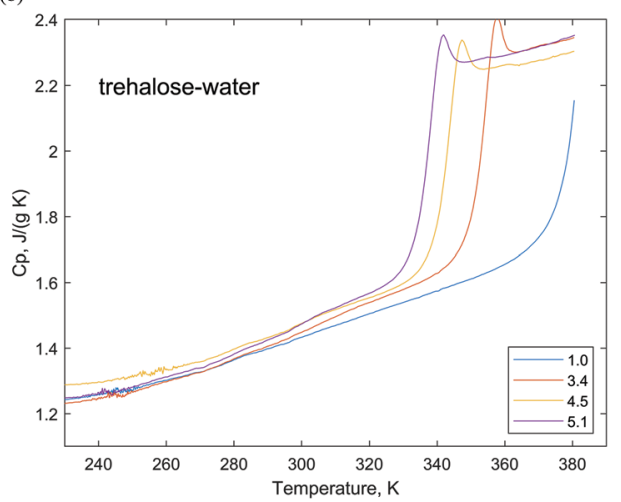

(c)

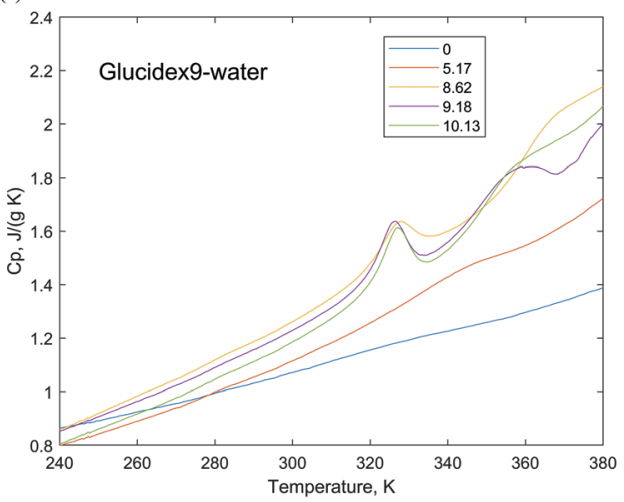

Fig. 5 Heat capacities of sucrose-water (a), trehalose-water (b) and Glucidex9-water (c) systems. Different colors correspond to different water contents (in wt\%).

sample of Glucidex9 shows no deviation from the baseline in the range of $240-380 \mathrm{~K}$. The glassy Glucidex9-water samples with water contents of $1.8-10.13 \mathrm{wt} \%$ show notable deviations from the baseline at about $320-340 \mathrm{~K}$, which corresponds to the sub- $T_{\mathrm{g}}$ transitions in starch-like materials followed by $T_{\mathrm{g}}$ at higher temperatures. The heat capacity dependencies of the samples with water contents higher than $10.13 \mathrm{wt} \%$ are presented in the ESI $\dagger$ (Fig. S4 and S5). They exhibit $T_{\mathrm{g}}^{\prime}$, ice melting and gelatinization thermal events.

For comparison with the sorption calorimetric experiments performed at $25{ }^{\circ} \mathrm{C}$, it is instructive to present the DSC heat capacity data as a function of water content at $25{ }^{\circ} \mathrm{C}$ (Fig. 6). 

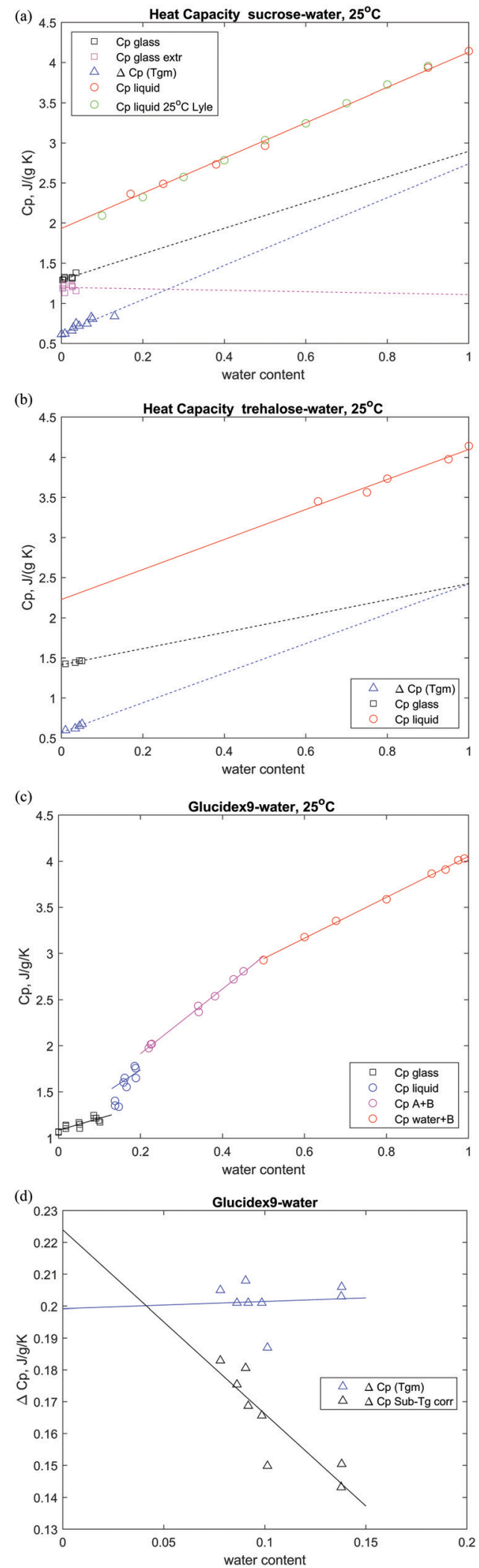

Fig. 6 The heat capacity and heat capacity changes in binary mixtures at $25{ }^{\circ} \mathrm{C}$ (unless stated otherwise in the legend). For sucrose, heat capacities in the liquid and glassy states are shown; the data for the glassy state marked as "extr" are obtained by extrapolation of the linear regime of the heat capacity curve before the glass transition; the literature data from the work of Lyle et al. ${ }^{43}$ are obtained by averaging the data at 20 and $30{ }^{\circ} \mathrm{C}$; the heat capacity change is for the glass transition temperature (a). For trehalose, the heat capacities in the liquid and glassy states are shown; the heat capacity change is for the glass transition temperature (b). For Glucidex9, the heat capacities in the glassy and liquid states, as well as the two-phase regions $(B+$ water, $A+B)$, are shown according to its phase diagram $^{25}$ (c). For Glucidex9, the heat capacity step is the direct measurements at $T_{\text {gmi }}$; data marked as "corr" are corrected for the sub- $T_{\mathrm{g}}$ endotherm (d).

The height of the heat capacity step at the glass transition temperature, $\Delta C_{p}\left(T_{\mathrm{gm}}\right)$, in the amorphous sucrose is presented as a function of water content in Fig. 6a (blue triangles). The baselines are taken before the sub- $T_{\mathrm{g}}$ event. The results indicate an increase of the heat capacity step with the increase of water content. All other data points in this figure are obtained at $25{ }^{\circ} \mathrm{C}$. The heat capacities of liquid sucrose solutions (red circles) cover a broad range of concentrations and are in good agreement with literature data (green circles). ${ }^{43}$ The heat capacities of the glassy mixtures are presented as black squares. Despite the rather narrow range of concentrations, an ascending trend is clearly seen (extrapolated with a black dashed line). A thorough examination of Fig. 5a suggests that this trend can be caused by the endothermic sub- $T_{\mathrm{g}}$ event rather than by the change of the main step size. In order to separate these two events, we calculated the extrapolated values of heat capacities in the glassy state that the system would show in case of the absence of sub- $T_{\mathrm{g}}$ (magenta squares). The extrapolated values were obtained by the linear extrapolation of the baseline measured in the range of $235-265 \mathrm{~K}$ to $298.15 \mathrm{~K}$. The results show that the main contribution to the increase in heat capacity in the glassy sucrose-water mixture was due to the sub- $T_{\mathrm{g}}$ event.

The heat capacities at $25{ }^{\circ} \mathrm{C}$ and the heat capacity change in the trehalose-water binary mixture are presented in Fig. 6b. All three presented parameters show a clear increase in water content.

The heat capacity of the Glucidex9-water mixture at $25{ }^{\circ} \mathrm{C}$ is presented in Fig. 6c. Four different regimes corresponding to different phase compositions of the system are clearly seen. The heat capacity of the glassy mixture (black squares) is in agreement with literature data. ${ }^{42}$ The second regime in the range of 13-18 wt\% corresponds to the liquid state (blue circles). Several points at the beginning of this regime (at 13.8-14 wt\%) probably correspond to the transition region between the glass and liquid states. It follows from the fact that the DSC heat capacity curves for these concentrations departed from the glassy baseline below $25{ }^{\circ} \mathrm{C}$ but the liquid state was not reached at this temperature. Therefore, the points at 13.8-14 wt\% were not taken into account for the calculation of the linear dependence of the liquid. To broaden the concentration range for linear fitting, the heat capacity of the pure liquid water was included in the calculations. Still, due to a very narrow concentration range of the liquid state in the Glucidexwater system, its heat capacity dependence on water content may be less accurate than that of other phases considered here.

Other concentration ranges (red and magenta circles) refer to two-phase regions: water $+\mathrm{B}$ and $\mathrm{A}+\mathrm{B}$, where $\mathrm{A}$ and $\mathrm{B}$ are the 
crystalline phases of maltodextrin according to its phase diagram. $^{25}$ The heat capacity change in the Glucidex9-water system directly measured at $T_{\text {gm }}$ is presented in Fig. 6d (blue triangles). The heat capacity change corrected for sub- $T_{\mathrm{g}}$ transitions (black triangles) will be discussed at the end of this section.

The presented data of heat capacity and heat capacity change in the two-component systems are approximated by linear fits. To highlight the contributions from the two components, linear equations can be represented in the following form. The heat capacity of the liquid binary mixture at $25{ }^{\circ} \mathrm{C}$ is:

$$
C_{p}^{1}=C_{p 1}^{0,1} \cdot w_{1}+C_{p 2}^{0,1} \cdot\left(1-w_{1}\right)
$$

where $C_{p 1}^{0,1}$ is the heat capacity of the liquid water, $C_{p 2}^{0,1}$ is the heat capacity of the liquid polymer, and $w_{1}$ is the mass fraction of water. The heat capacity of the glassy binary mixture at $25{ }^{\circ} \mathrm{C}$ is:

$$
C_{p}^{\mathrm{g}}=C_{p 1}^{\varnothing, \mathrm{g}} \cdot w_{1}+C_{p 2}^{0, \mathrm{~g}} \cdot\left(1-w_{1}\right)
$$

where $C_{p 1}^{\varnothing, g}$ is the apparent heat capacity of the glassy water, and $C_{p 2}^{0, g}$ is the heat capacity of the glassy carbohydrate.

Subtracting eqn (4) and (5) gives an expression for $\Delta C_{p}$ :

$$
\Delta C_{p}=\Delta C_{p 1}^{\varnothing} \cdot w_{1}+\Delta C_{p 2}^{0} \cdot\left(1-w_{1}\right)
$$

where $\Delta C_{p 1}^{\varnothing}$ is the apparent heat capacity change of water, and $\Delta C_{p 2}^{0}$ is the heat capacity change of the carbohydrate.

The $\Delta C_{p i}^{0}$ values for sucrose obtained in two different ways $\left(\Delta C_{p i}^{0}\right.$ measured at $T_{\mathrm{gm}}$ and $C_{p i}^{0,1}-C_{p i}^{0, \mathrm{~g}}$ extr measured at $25^{\circ} \mathrm{C}$ ) are in reasonable agreement (see Table 4) and the second data set is chosen for comparison with the sorption calorimetry results in Table 3. In the case of trehalose, $C_{p i}^{0,1}-C_{p i}^{0, g}$ values of the heat capacity step were chosen for comparison with the sorption calorimetry results in Table 3 .

The heat capacity changes of Glucidex9 and Glucidex12 calculated as a difference between the linear approximations for the liquid and glassy states $\left(C_{p i}^{0,1}-C_{p i}^{0, \mathrm{~g}}\right.$ at $25{ }^{\circ} \mathrm{C}$ in Table 5) show too small values for the dry polymers. The reason for this

Table 4 The heat capacity and heat capacity step in the binary mixtures of sucrose-water and trehalose-water systems in $\mathrm{J} \mathrm{g}^{-1} \mathrm{~K}^{-1}$ at $25^{\circ} \mathrm{C}$ mea-

\begin{tabular}{|c|c|c|c|c|}
\hline & \multicolumn{2}{|l|}{ Sucrose } & \multicolumn{2}{|l|}{ Trehalose } \\
\hline & Water (1) & Sucrose (2) & Water (1) & Trehalose (2) \\
\hline$C_{p i}^{0,1}$ liquid & 4.13 & 1.93 & 4.10 & 2.22 \\
\hline$C_{p i}^{0, \mathrm{~g}}$ glass & $2.90^{\varnothing}$ & 1.29 & $2.42^{\varnothing}$ & 1.41 \\
\hline$C_{p l}^{0, g}$ glass extr ${ }^{a}$ & $1.11^{\varnothing}$ & 1.20 & - & - \\
\hline$C_{p i}^{0,1}-C_{p i}^{0, g}$ & $1.23^{\varnothing}$ & 0.64 & $1.67^{\varnothing}$ & 0.81 \\
\hline$C_{p i}^{0,1}-C_{p i}^{0, \mathrm{~g}} \operatorname{extr}^{a}$ & $\underline{3.02} \underline{\underline{ }}^{\varnothing}$ & $\underline{0.7} \underline{3}$ & - & - \\
\hline$\Delta C_{p i}^{0}$ at $T_{\mathrm{gm}}{ }^{b}$ & $2.74 \varnothing$ & $\overline{0.62}$ & $2.42^{\varnothing}$ & 0.57 \\
\hline
\end{tabular}
sured by DSC. The underlined values are used in Table 3 for comparison with sorption calorimetry

$\varnothing$ - apparent values (extrapolated to pure water). ${ }^{a}$ Extrapolated values the values obtained by linear extrapolation of the baseline in the range of $235-265 \mathrm{~K}$ to $298.15 \mathrm{~K} .{ }^{b}$ Direct measurement - values evaluated using METTLER TOLEDO software according to ISO standard, baselines were tangent lines before the sub- $T_{\mathrm{g}}$ deviation. These values are measured at the glass transition temperature of the mixture.
Table 5 The heat capacity and heat capacity step of the Glucidex9-water and Gluciedex12-water systems in $\mathrm{J} \mathrm{g}^{-1} \mathrm{~K}^{-1}$ at $25^{\circ} \mathrm{C}$ measured by DSC.

\begin{tabular}{|c|c|c|c|c|}
\hline & \multicolumn{2}{|c|}{ Glucidex9 } & \multicolumn{2}{|c|}{ Glucidex12 } \\
\hline & $\begin{array}{l}\text { Water } \\
\text { (1) }\end{array}$ & $\begin{array}{l}\text { Glucidex9 } \\
\text { (2) }\end{array}$ & $\begin{array}{l}\text { Water } \\
\text { (1) }\end{array}$ & $\begin{array}{l}\text { Glucidex12 } \\
\text { (2) }\end{array}$ \\
\hline$\overline{C_{p i}^{0,1} \text { liquid }}$ & 4.06 & $1.16^{c}$ & 4.00 & $1.12^{c}$ \\
\hline$C_{p i}^{0, \mathrm{~g}}$ glass & $2.37^{\varnothing}$ & 1.08 & $2.33^{\varnothing}$ & 1.02 \\
\hline$C_{p i}^{0,1}-C_{p i}^{0, g}$ at $25^{\circ} \mathrm{C}$ & $1.70^{\varnothing}$ & $0.08^{c}$ & $1.67^{\varnothing}$ & $0.10^{c}$ \\
\hline$\Delta C_{p i}^{0}$ at $T_{\mathrm{gm}}{ }^{a}$ & $0.22^{\varnothing}$ & 0.20 & $0.24^{\varnothing}$ & 0.23 \\
\hline$\Delta C_{p i}^{0}$ sub- $T_{\mathrm{g}}$ corrected $^{b}$ & $-0.3 \underline{5}^{\varnothing}$ & $\underline{0.22}$ & $-0.31^{\varnothing}$ & $\underline{0.22}$ \\
\hline
\end{tabular}
The underlined values are used in Table 3 for comparison with sorption calorimetry

$\varnothing$ - apparent values. ${ }^{a}$ Direct measurement - values evaluated using METTLER TOLEDO software according to ISO standard, baselines are taken above the sub- $T_{\mathrm{g}} .{ }^{b}$ Correction on sub- $T_{\mathrm{g}}$ according to eqn (8). ${ }^{c}$ Accuracy of these values can be affected by the narrow concentration range in the liquid state.

is the low accuracy for the linear approximation in the liquid state due to a very narrow concentration range. Therefore, the $\Delta C_{p}$ data obtained directly at $T_{\mathrm{gm}}$ in a broader concentration range can be considered more appropriate for monitoring the concentration dependence. The apparent values of heat capacity change for water $\left(\Delta C_{p 1}^{\varnothing}\right)$ obtained from these data are much lower compared to $\Delta C_{p 1}^{0}$ for pure water. Still, they are not negative (as one could expect from the comparison with the sorption calorimetry data). To explain this discrepancy, we recall that the glass transition in eqn (4) is approximated as a single step, not dependent on temperature. A rigorous equation for the calculation of hydration enthalpy ${ }^{28}$ includes the integration of heat capacity with respect to temperature rather than the multiplication of heat capacity changes by the temperature differences. In such integration, not only the heat capacity step but also the sub- $T_{\mathrm{g}}$ peak should be taken into account (Fig. 7). Effectively, the presence of the sub- $T_{\mathrm{g}}$ peak corresponds to the increase of the heat capacity in the glassy state (red dashed line in Fig. 7) and therefore the decrease of the heat capacity step.

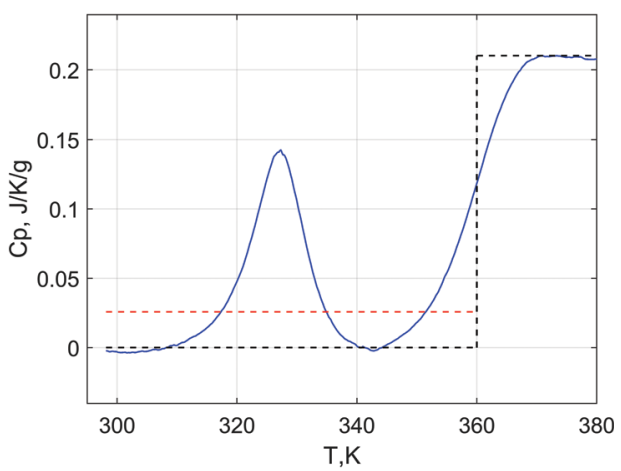

Fig. 7 The heat capacity of the maltodextrin-water mixture $(8.62$ wt\% water) after subtracting the baseline (blue curve). The black dashed line shows the approximation of the glass transition as a step. The red dashed line shows the position of the baseline corresponding to the glassy state that takes into account the area of the sub- $T_{\mathrm{g}}$ endotherm. 
In order to take this into account, we implemented a correction of the heat capacity change:

$$
\Delta C_{P \mathrm{corr}}^{0}=\Delta C_{P}^{0}-\frac{\Delta H_{\mathrm{sub}}^{\circ} \cdot w_{1}}{T_{\mathrm{gm}}-T}
$$

where $\Delta H_{\text {sub }}^{\circ}$ is the slope of the enthalpy of the sub- $T_{\mathrm{g}}$ peak with respect to $w_{1}(\mathrm{~S} 6, \mathrm{~S} 7)$.

The corrected data are shown in Table 5 (last row) and are chosen for comparison with the sorption calorimetry results (Table 3).

\subsection{Differences in water sorption behavior between disaccharides and maltodextrins}

3.4.1. Sucrose vs. trehalose. In literature, one can find discussion about the superior properties of trehalose in comparison with sucrose $\mathrm{e}^{44}$ for the preservation of proteins and bacteria. When discussing the preservation and stabilization mechanisms, the cases of liquid and solid formulations should be considered separately. In liquid solutions, it is relevant to understand if the protein surface is preferentially in contact with water or the carbohydrate. To describe this, two models were proposed: the water replacement $\operatorname{model}^{45}$ and the preferential hydration model. ${ }^{46}$ Recently, it has been shown that in concentrated liquid solutions, the stabilization is due to the effect of trehalose on the dynamics of hydration, rather than the direct protein-sugar interactions. ${ }^{47}$ In solid formulations, where almost all water is removed and the protein-sugar interactions are inevitable, the stabilization mechanisms are different. For solid-state amorphous formulations, the glass transition temperature of the carbohydrate matrix is important ${ }^{48,49}$ since for best protection, the storage temperature should be at least $20{ }^{\circ} \mathrm{C}$ below the $T_{\mathrm{g}} \cdot{ }^{1}$ Moreover, the hydrophilicity of the carbohydrate and its propensity to crystallize $^{48,50}$ influence the stability. Furthermore, the structural features and energies of protein-sugar interactions play a role. ${ }^{51}$ Finally, the molecular motions in the vitrified carbohydrate matrix ( $\beta$-relaxations of water and carbohydrates) are important for protein stability. ${ }^{52,53}$

Based on the results presented in this work, the differences in properties of the two carbohydrates can be illustrated in terms of the thermodynamics of hydration. For a better comparison, we combine the experimental data for sucrose and trehalose, as shown in Fig. 8. In the sorption calorimetry experiments, we observe the water-induced crystallization of sucrose anhydrous crystals as well as trehalose dihydrate. In the case of slow scan rate, the crystallization in the sucrose-water system occurs at the water content of $5.5 \mathrm{wt} \%$ and $a_{\mathrm{w}}=0.3$, whereas in the trehalose-water system, the dihydrate crystallizes at $9.5-10 \mathrm{wt} \%$ and $a_{\mathrm{w}}=0.45-0.5$. Accordingly, the trehalose-water system undergoes water-induced crystallization at higher water content and the glassy state accumulates a higher amount of water prior to crystallization. In this way, the trehalose-water system has higher water sorption capacity or "water stability" with respect to crystallization.
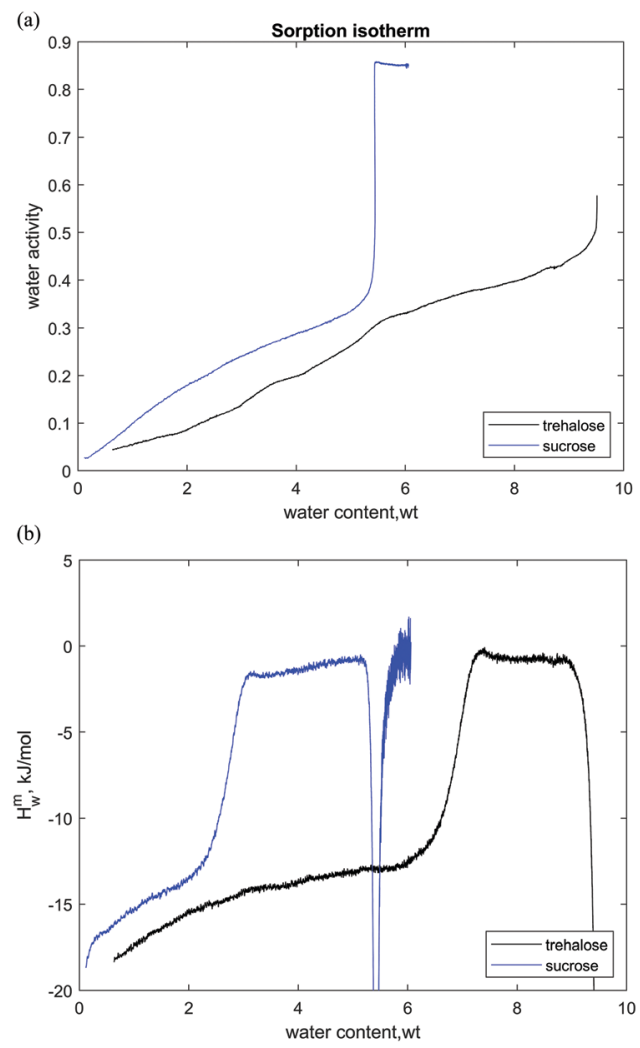

Fig. 8 Water sorption isotherms of sucrose and trehalose (a) and partial molar enthalpies of mixing of water (b). Slow scan rates, $25^{\circ} \mathrm{C}$.

A contrast between the two disaccharides is also seen in the coordinates of the water-induced glass transition at $25{ }^{\circ} \mathrm{C}$. The sucrose-water system undergoes a water-induced glass transition at $2.5-3 \mathrm{wt} \%\left(a_{\mathrm{w}}=0.2-0.25\right)$, while trehalose at $7 \mathrm{wt} \%\left(a_{\mathrm{w}}=0.35\right)$. This is due to the fact that the glass transition temperature of the dry sucrose $\left(70{ }^{\circ} \mathrm{C}\right)$ is lower than that of trehalose $\left(120{ }^{\circ} \mathrm{C}\right)$ as mentioned above.

The better water sorption capacity of trehalose can be explained based on the hydration enthalpy data (Fig. 8b). The water activity in the glassy state is dependent on the hydration enthalpy as follows:

$$
\frac{\mathrm{d} \ln a_{1}}{\mathrm{~d} T}=-\frac{H_{1}^{m, g}}{R T^{2}}
$$

In order to get the value of water activity at temperature $T$, one needs to integrate the right-hand side of eqn (9) from $T_{\mathrm{gm}}$ down to $T$. Lower (more exothermic) values of enthalpy will result in lower activity values. In the case of trehalose, the enthalpy values are not only more exothermic, but also the integration is done at a broader range of temperatures (since trehalose has a higher $T_{\mathrm{g}}$ value), which further decreases the water activity. A quantitative description of the calculation of water activity in glassy materials will be presented elsewhere. ${ }^{54}$

3.4.2. Disaccharides vs. carbohydrate polymers. As it was shown in the previous sections, disaccharides and carbohydrate polymers have different thermodynamic properties related to water sorption. Below, we will summarize the experimentally 
observed differences and try to propose a molecular-level explanation.

The heat capacity step $\left(\Delta C_{p 2}\right)$ in the case of small sugars is several times higher than that in maltodextrins according to both DSC and sorption calorimetry data (Table 3). Interestingly, the main difference arises from the heat capacity in the liquid state $\left(C_{p 2}^{0,1}\right)$, where the heat capacities of liquid sucrose and trehalose are higher than those of maltodextrins (see Tables 4 and 5). The difference in heat capacities in the glassy state is less pronounced. In terms of molecular motions, this can be explained by the fact that there are more degrees of freedom in disaccharides than in polymers, and their contributions to heat capacities are more pronounced in the liquid state, while being "frozen" in the glassy state.

In the enthalpy of mixing plot, the glass transition step $\left(\Delta H_{1}^{m}\right)$ in the case of sugars is higher than that in maltodextrins. This is in good correlation with the heat capacity step of dry substances and matches the predictions of the "constant enthalpy" models.

The glass transition-related heat capacity changes for water $\left(\Delta C_{p 1}^{\varnothing}\right)$ are positive for the disaccharides (Table 3) both in DSC and sorption calorimetry measurements. ${ }^{38}$ Moreover, the values of heat capacity of glassy water $\left(C_{p 1}^{\varnothing, \mathrm{g}}\right)$ estimated from the long linear extrapolation of the sucrose-water and trehalose-water systems (Table 4 ) are in reasonable agreement with the heat capacity of glassy water of $2.26 \mathrm{~J} \mathrm{~g}^{-1} \mathrm{~K}^{-1}$ at $300 \mathrm{~K}$ calculated by Pyda. ${ }^{20}$

In the case of maltodextrins, the situation is different. The apparent change of heat capacity of water is negative or close to 0 . This can be interpreted as water molecules undergo a glass transition together with the sucrose and trehalose matrices, while in maltodextrins, they remain mobile.

For the calculations of $\Delta C_{p 1}^{\varnothing}$ for polymers, an additional factor plays a role. The maltodextrins have a sub- $T_{\mathrm{g}}$ endotherm in the form of a peak that has a substantial area (additional enthalpy) that affects the calculation of the enthalpy using eqn (4). The presence of a peak is equivalent to the increase of the baseline and the decrease of the heat capacity step as illustrated in Fig. 7.

The difference in the thermodynamic properties of water in the glassy matrix of small sugars and polymers also correlated with its dynamical properties. The water diffusion coefficient of the sucrose-water mixtures at a water activity of 0.2 (slightly below glass transition) was measured by the Raman isotope tracer method $\left(10^{-16} \mathrm{~m}^{2} \mathrm{~s}^{-1}\right)$. The water diffusion coefficient of the maltodextrin-water mixtures was estimated to be $10^{-13} \mathrm{~m}^{2} \mathrm{~s}^{-1} \cdot{ }^{34}$ Water molecules move orders of magnitude faster in the polymer-water system than in the sucrose-water system, which supports the idea that in the polymer matrix, water molecules do not exhibit properties typical for the glassy state.

3.4.3. Implication of the results for pharmaceutical formulations. Amorphous disaccharides and maltodextrins are used in pharmaceutical and food industries as excipients and for cryopreservation of proteins and microorganisms. Glassy states are essential for the stabilization of biologics in dry formulations, obtained by freeze-drying or spray-drying. However, the exact mechanisms are still not fully understood. There are two main hypotheses for protein stabilization by amorphous sugars: the water replacement theory and the dynamic theory.

The concept of the water replacement theory is as follows. ${ }^{2,55,56}$ Upon dehydration, water molecules are replaced by sugar molecules, which form hydrogen bonds with the protein. ${ }^{57}$ The hydrogen bonds are essential to maintain the three-dimensional structure of the protein, and hence the sugar molecules can support the protein structure by replacing water in these interactions. The basis for the dynamic theory is the restriction of the molecular movements of the protein, as well as the dilution effect of the sugar matrix, ${ }^{58}$ which reduces the direct protein-protein interactions. Within the frame of this theory, the molecular movements of the protein are "frozen" by incorporation into the glassy matrix.

The physical stability of amorphous formulations is determined by the glass transition temperature. The glass transition temperature is related to the overall molecular motions of the amorphous matrix. In particular, the higher the glass transition temperature, the lower the molecular mobility of the polymer or carbohydrate. The glass transition temperature does, however, not tell the whole story. The heat capacity is almost equally important for understanding the dynamics of the complex binary mixture. The fact that the heat capacity change is twice as high as that in disaccharides compared to polymers shows that the mobility change in smaller molecules is more pronounced at the glass transition temperature. The most interesting observation comes from the fact that the heat capacity change of water in polymers is much lower than that in disaccharides. This indicates that the mobility of water is uncoupled from the mobility of polymers, while in disaccharides, water changes its mobility together with the whole system.

This behavior can be rationalized in terms of free volume approaches. When water molecules penetrate through the glassy matrix during the hydration process, the density of the matrix shows a strong influence on this process. The true density is the mass of a particle divided by its volume, excluding any macroscopic cavities. Based on molecular considerations, the density of a polysaccharide is expected to be higher than the density of a disaccharide. Nevertheless, experimental data for densities in the glassy state do not show a significant difference between the two classes of substances. For example, the true density of the amorphous sucrose was found to be $1.518 \mathrm{~g} \mathrm{~cm}^{-3}$, trehalose $1.505 \mathrm{~g} \mathrm{~cm}{ }^{-3},{ }^{59}$ and maltodextrin with a DE-12 $1.5091 \mathrm{~g} \mathrm{~cm}^{-3} \cdot{ }^{60}$ The evolution of the density of the glassy carbohydrates upon addition of water is, however, different. The density of the maltose-water mixtures decreases upon hydration. ${ }^{61}$ This correlation is expected since the density of the amorphous disaccharides is higher than that of water. The opposite trend (density increases) was shown for the maltodextrin-water mixture ${ }^{60}$ at low water content. Even though the density of liquid water is lower than the density of maltodextrin, the addition of small amounts of water increases the density of the system. This suggests that the polymer matrix has a higher free volume, which is filled with water molecules. This also leads to a higher exothermic effect of the enthalpy of mixing with water (Fig. 1 and 2). The free volume in 
the glassy matrix allows more space for the diffusion of small molecules. As a result, in maltodextrins, water moves three orders of magnitude faster than in disaccharides since there is more available space in the former.

The properties of the glassy matrix affect the penetrant behavior. For best preservation of proteins and probiotics, the selection of carbohydrate excipients should be optimized to provide good interactions with the protein while at the same time providing a physically stable matrix, i.e., has a sufficiently high glass transition temperature. One of the most important parameters to consider here is the behavior of residual water in the glassy matrix. The results presented in this work can help in designing solid-state pharmaceutical formulations with increased stability.

\section{Conclusions}

Here we present the sorption calorimetry and DSC studies of interactions between water and amorphous sucrose, trehalose and maltodextrins. We conclude that:

- The hydration enthalpies of glassy sucrose, trehalose and maltodextrins are exothermic.

- The water-induced glass transition is seen as a step on the enthalpy plot, and the step is higher for small molecules.

- The partial molar enthalpy of mixing of water in slow experiments is about $-18 \mathrm{~kJ} \mathrm{~mol}^{-1}$ for all the studied carbohydrates, but less exothermic in the case for small molecules at fast hydration scan rates.

- For disaccharides, the water-induced glass transition and crystallization at $25{ }^{\circ} \mathrm{C}$ shift to lower hydration levels at slow scan rates, while for maltodextrins, this is not observed.

- By measuring the heat capacities of disaccharides and maltodextrins as a function of water content, we separated the contributions of carbohydrates and water to the total heat capacities of the mixtures.

- The heat capacity changes calculated by the fitting of the hydration enthalpy data for disaccharides are in good agreement with the heat capacity data obtained by DSC.

- The heat capacity changes of water in glassy maltodextrins evaluated from the sorption calorimetry data are negative, which can be explained by the effect of sub- $T_{\mathrm{g}}$ transitions in maltodextrins.

- The DSC heat capacity curves of maltodextrin show a sub$T_{\mathrm{g}}$ transition in the form of a clear peak, amorphous sucrose has a sub- $T_{\mathrm{g}}$ endotherm as a shift of the baseline before the glass transition, and for trehalose no sub- $T_{\mathrm{g}}$ events were observed.

- The relaxation phenomena such as enthalpy relaxation and sub- $T_{\mathrm{g}}$ of the amorphous polymers affect the enthalpy of mixing with water.

- The data described above suggest that in disaccharides, the glass transition of water is more pronounced, while in maltodextrin it is less coupled to the carbohydrate matrix.

\section{Conflicts of interest}

There are no conflicts of interest to declare.

\section{Acknowledgements}

This research was funded by the Swedish Governmental Agency for Innovation Systems (VINNOVA) and was carried out within the competence center NextBioForm (grant number 2018-04730).

\section{References}

1 L. Chang and M. J. Pikal, Mechanisms of Protein Stabilization in the Solid State, J. Pharm. Sci., 2009, 98(9), 2886-2908.

$2 \mathrm{~W}$. Wang, Lyophilization and development of solid protein pharmaceuticals, Int. J. Pharm., 2000, 203(1-2), 1-60.

3 S. P. Duddu and P. R. Dal Monte, Effect of Glass Transition Temperature on the Stability of Lyophilized Formulations Containing a Chimeric Therapeutic Monoclonal Antibody., Pharm. Res., 1997, 14, 591-595.

4 B. C. Hancock and G. Zografi, Characteristics and Significance of the Amorphous State in Pharmaceutical Systems, J. Pharm. Sci., 1997, 86(1), 1-12.

5 T. Arakawa, et al., Factors affecting short-term and longterm stabilities of proteins, Adv. Drug Delivery Rev., 2001, 46, 307-326.

6 M. Gordon and J. S. Taylor, Ideal copolymers and the second-order transitions of synthetic rubbers. I. noncrystalline copolymers, J. Appl. Chem., 1952, 2(9), 493-500.

7 Y. H. Roos and M. Karel, Water and Molecular-Weight Effects on Glass Transitions in Amorphous Carbohydrates and Carbohydrate Solutions, J. Food Sci., 1991, 56(6), 1676-1681.

8 K. Elamin, et al., Different behavior of water in confined solutions of high and low solute concentrations, Phys. Chem. Chem. Phys., 2013, 15(42), 18437-18444.

$9 \mathrm{~J}$. Swenson, et al., Why is there no clear glass transition of confined water?, Chem. Phys., 2013, 424, 20-25.

10 L. Mascia, et al., Antiplasticization of Polymer Materials: Structural Aspects and Effects on Mechanical and DiffusionControlled Properties, Polymers, 2020, $12(4), 769$.

11 V. Kocherbitov, et al., Hydration of microcrystalline cellulose and milled cellulose studied by sorption calorimetry, J. Phys. Chem. B, 2008, 112(12), 3728-3734.

12 D. Lechuga-Ballesteros, A. Bakri and D. P. Miller, Microcalorimetric measurement of the interactions between water vapor and amorphous pharmaceutical solids, Pharm. Res., 2003, 20(2), 308-318.

13 J. S. Vrentas and C. M. Vrentas, Sorption in Glassy-Polymers, Macromolecules, 1991, 24(9), 2404-2412.

14 B. C. Hancock and G. Zografi, The Use of Solution Theories for Predicting Water-Vapor Absorption by Amorphous Pharmaceutical Solids - a Test of the Flory-Huggins and Vrentas Models, Pharm. Res., 1993, 10(9), 1262-1267.

15 R. G. M. van der Sman and M. B. J. Meinders, Prediction of the state diagram of starch water mixtures using the FloryHuggins free volume theory, Soft Matter, 2011, 7, 429-442. 
16 J. Zhang and G. Zografi, The Relationship Between "BET"and "Free Volume"-Derived Parameters for Water Vapor Absorption into Amorphous Solids, J. Pharm. Sci., 2000, 89(8), 1063-1072.

$17 \mathrm{~J}$. Zhang and G. Zografi, Water Vapor Absorption Into Amorphous Sucrose-Poly(Vinyl Pyrrolidone) and TrehalosePoly(Vinyl Pyrrolidone) Mixtures, J. Pharm. Sci., 2001, 90(9), 1375-1385.

18 S. L. Shamblin, et al., Characterization of the Time Scales of Molecular Motion in Pharmaceutically Important Glasses, J. Phys. Chem. B, 1999, 103, 4113-4121.

19 A. Magon, et al., Heat capacity and transition behavior of sucrose by standard, fast scanning and temperaturemodulated calorimetry, Thermochim. Acta, 2014, 589, 183-196.

20 M. Pyda, Conformational Contribution to the Heat Capacity of the Starch and Water System, J. Polym. Sci., Part B: Polym. Phys., 2001, 39(23), 3038-3054.

21 D. P. Miller and J. J. de Pablo, Calorimetric Solution Properties of Simple Saccharides and Their Significance for the Stabilization of Biological Structure and Function, J. Phys. Chem. B, 2000, 104, 8876-8883.

22 L. Wadsö and N. Markova, A method to simultaneously determine sorption isotherms and sorption enthalpies with a double twin microcalorimeter, Rev. Sci. Instrum., 2002, 73(7), 2743-2754.

23 Y. Znamenskaya, et al., Effect of hydration on structural and thermodynamic properties of pig gastric and bovine submaxillary gland mucins, J. Phys. Chem. B, 2012, 116(16), 5047-5055.

24 J. E. Cathrine Albèr, P. Falkman and V. Kocherbitov, Hydration of Hyaluronan: Effects on Structural and Thermodynamic Properties, J. Phys. Chem. B, 2015, 119, 4211-4219.

25 J. Carlstedt, J. Wojtasz, P. Fyhr and V. Kocherbitov, Hydration and the phase diagram of acid hydrolyzed potato starch, Carbohydr. Polym., 2014, 112, 569-577.

26 V. Kocherbitov, T. Arnebrant and O. Soderman, Lysozymewater interactions studied by sorption calorimetry, J. Phys. Chem. B, 2004, 108(49), 19036-19042.

27 V. Kocherbitov and O. Soderman, Glassy Crystalline State and Water Sorption of Alkyl Maltosides, Langmuir, 2004, 20, 3056-3061.

28 V. Kocherbitov and I. Argatov, Enthalpy of sorption by glassy polymers, Polymer, 2019, 174, 33-37.

29 L. Greenspan, Humidity fixed-points of binary saturated aqueous-solutions, J. Res. Natl. Bur. Stand., Sect. A, 1977, 81(1), 89-96.

30 V. Kocherbitov, A new formula for accurate calculation of water activity in sorption calorimetric experiments, Thermochim. Acta, 2004, 414(1), 43-45.

31 V. Kocherbitov, On the calculation of thermodynamic parameters in sorption calorimetric experiments, J. Chem. Thermodyn., 2021, 152, 106264.

32 B. Makower and W. B. Dye, Equilibrium Moisture Content and Crystallization of Amorphous Sucrose and Glucose, J. Agric. Food Chem., 1956, 4(1), 72-77.
33 H. C. Price, et al., Quantifying water diffusion in highviscosity and glassy aqueous solutions using a Raman isotope tracer method, Atmos. Chem. Phys., 2014, 14(8), 3817-3830.

34 M. Räderer, A. Besson and K. Sommer, A thin film dryer approach for the determination of water diffusion coefficients in viscous products, Chem. Eng. J., 2002, 86, 185-191.

35 G. P. Johari, G. Astl and E. Mayer, Enthalpy Relaxation of Glassy Water, J. Chem. Phys., 1990, 92(1), 809-810.

36 T. Chen, A. Fowler and M. Toner, Literature review: supplemented phase diagram of the trehalose-water binary mixture, Cryobiology, 2000, 40(3), 277-282.

37 J. Swenson, Possible relations between supercooled and glassy confined water and amorphous bulk ice, Phys. Chem. Chem. Phys., 2018, 20(48), 30095-30103.

38 S. Masayasu, S. Hiroshi and S. Syûzô, Calorimetric Study of the Glassy State. IV. Heat Capacities of Glassy Water and Cubic Ice., Bull. Chem. Soc. Jpn., 1968, 41(11), 2591-2599.

39 H. J. Thiewes and P. A. M. Steeneken, The glass transition and the sub-T, endotherm of amorphous and native potato starch at low moisture content, Carbohydr. Polym., 1996, 32, 123-130.

40 J. W. Lee, L. C. Thomas and S. J. Schmidt, Effects of Heating Conditions on the Glass Transition Parameters of Amorphous Sucrose Produced by Melt-Quenching, J. Agric. Food Chem., 2011, 59(7), 3311-3319.

41 E. Tombari and G. P. Johari, Endothermic Effects on Heating Physically Aged Sucrose Glasses and the Clausius Theorem Violation in Glass Thermodynamics, J. Phys. Chem. B, 2020, 124, 2017-2028.

42 T. R. Noel and S. G. Ring, A study of the heat capacity of starch/water mixtures, Carbohydr. Res., 1992, 227, 203-213.

43 O. Lyle, Technology for Sugar Refinery Workers, Chapman \& Hall pub, UK, London, 1957.

44 S. Ohtake and Y. J. Wang, Trehalose: Current use and future applications, J. Pharm. Sci., 2011, 100(6), 2020-2053.

45 T. Arakawa, Y. Kita and J. F. Carpenter, Protein-Solvent Interactions in Pharmaceutical Formulations, Pharm. Res., 1991, 8(3), 285-291.

46 S. N. Timasheff, Protein Hydration, Thermodynamic Binding, and Preferential Hydration, Biochemistry, 2002, 41(46), 13473-13482.

47 C. Olsson, et al., Mechanism of Trehalose-Induced Protein Stabilization from Neutron Scattering and Modeling. The, J. Phys. Chem. B, 2019, 123(17), 3679-3687.

48 L. M. Crowe, D. S. Reid and J. H. Crowe, Is trehalose special for preserving dry biomaterials?, Biophys. J., 1996, 71(4), 2087-2093.

49 N. Grasmeijer, et al., Unraveling protein stabilization mechanisms: vitrification and water replacement in a glass transition temperature controlled system, Biochim. Biophys. Acta, 2013, 1834(4), 763-769.

50 F. Librizzi, E. Vitrano and L. Cordone, Dehydration and Crystallization of Trehalose and Sucrose Glasses Containing Carbonmonoxy-Myoglobin, Biophys. J., 1999, 76(5), 2727-2734. 
51 S. Giuffrida, et al., Proteins in amorphous saccharide matrices: Structural and dynamical insights on bioprotection, Eur. Phys. J. E: Soft Matter Biol. Phys., 2013, 36(7), 79.

52 C. Olsson, R. Zangana and J. Swenson, Stabilization of proteins embedded in sugars and water as studied by dielectric spectroscopy, Phys. Chem. Chem. Phys., 2020, 22(37), 21197-21207.

53 T. Starciuc, et al., Trehalose or Sucrose: Which of the Two Should be Used for Stabilizing Proteins in the Solid State? A Dilemma Investigated by In Situ Micro-Raman and Dielectric Relaxation Spectroscopies During and After FreezeDrying, J. Pharm. Sci., 2020, 109(1), 496-504.

54 V. Kocherbitov and I. Argatov, A thermodynamic theory of sorption in glassy polymers, submitted for publication.

55 M. A. Mensink, et al., How sugars protect proteins in the solid state and during drying (review): mechanisms of stabilization in relation to stress conditions, Eur. J. Pharm. Biopharm., 2017, 114, 288-295.
56 F. M. Richards, Protein stability: still an unsolved problem, Cell. Mol. Life Sci., 1997, 53(10), 790-802.

57 J. H. Crowe, A. F. A. Hoekstra and L. M. Crowe, Anhydrobiosis., Annu. Rev. Physiol., 1992, 54(1), 579-599.

58 R. V. Mannige, Dynamic New World: Refining Our View of Protein Structure, Function and Evolution, Proteomes, 2014, 2(1), 128-153.

59 K. Imamura, et al., True Density Analysis of a Freeze-Dried Amorphous Sugar Matrix, J. Pharm. Sci., 2008, 97(7), 2789-2797.

60 D. Kilburn, et al., Water in glassy carbohydrates: Opening it up at the nanolevel, J. Phys. Chem. B, 2004, 108(33), 12436-12441.

61 D. Lourdin, P. Colonna and S. G. Ring, Volumetric behaviour of maltose-water, maltose-glycerol and starch-sorbitol-water systems mixtures in relation to structural relaxation, Carbohydr. Res., 2003, 338(24), 2883-2887. 